\title{
Source wavelet estimation in FWI context
}

\author{
Felipe Timóteo da Costa, Edmarley Costa Ramos, Marco Antonio Cetale Santos, GISIS/DOT-UFF, Brazil
}

Copyright 2019, SBGf - Sociedade Brasileira de Geofísica.

This paper was prepared for presentation at the $16^{\text {th }}$ International Congress of the Brazilian Geophysical Society, held in Rio de Janeiro, Brazil, August 19-22, 2019.

Contents of this paper were reviewed by the Technical Committee of the $16^{\text {th }}$ International Congress of The Brazilian Geophysical Society and do not necessarily represent any position of the SBGf, its officers or members. Electronic reproduction or storage of any part of this paper for commercial purposes without the written consent of The Brazilian Geophysical Society is prohibited.

\section{Abstract}

In time domain acoustic full waveform inversion (FWI) the source wavelet needs to be estimated for each frequency due the multiscale approach. The same adjoint method used to find the velocity model perturbation in FWI workflow can be applied to estimate the source wavelet. We carried out a 2D synthetic experiment simulating an ocean bottom cable (OBC) acquisition to generate the observed data used in both wavelet inversion and velocity model inversion. The observed data was filtered in different ranges of frequency to prepare it for the FWI workflow. The inversion problem in which the source wavelet is estimated uses the 1D wave equation to generate the calculated data. Because of it, the estimated wavelet needed to be rescaled to compensate the difference between 1D and 2D wave equation modeling. After evaluation of the objective function (cost function, error function), its gradient was obtained by the adjoint method. And the gradient direction was used to minimize the objective function in an iterative nonlinear optimization problem. The nonlinear optimization problem was solved by the steepest descent method. This strategy allowed to find an inverted source wavelet that produced consistent results in generating the $2 \mathrm{D}$ calculated data. Furthermore, the estimated source wavelet was used in acoustic FWI workflow to estimate a velocity model. The results indicate that the velocity inversion using the estimated source wavelets will converge. As long as, the main structures of the true velocity model, used to generate the observed data, are identified in the inverted velocity model. With this methodology was possible to perform a more realistic synthetic experiment.

\section{Introduction}

The wavelet estimation is an important stage in full waveform inversion (FWI) method. The estimative of appropriated wavelet will permit to generate a calculated data that will produce a more accurate fit with observed data. Resulting in better convergence proprieties of the optimization method used to solve the nonlinear inverse problem. In time domain FWI problem, the estimative of source wavelet need to be separated into different ranges of frequency because of multiscale strategy. This approach will mitigate the cycle skipping problem (Bunks et al., 1995;
Pratt, 1999; Virieux and Operto, 2009; Brossier et al., 2009).

There are many different ways to extract the source wavelet from observed data (Sears et al., 2010). Most of them employ the convolutional model to extract the source wavelet from seismic sections. Those methods typically require a series of reflectivity obtained from welllog information. When there is no well-log information, the reflectivity series is assumed to be an uncorrelated random variable (Wang, 2016). In FWI synthetic tests, the source wavelet estimation is harder because we would need a stack seismic section to use the convolution-based source wavelet estimation.

In order to avoid any kind of seismic processing in synthetic seismic experiments, we estimate the source wavelet using only the 1D wave equation and 2D pre-stack dataset. We solve a nonlinear inversion problem (Nocedal and Wright, 2006) in which the source wavelet is the parameter to be found by the optimization method. This problem was solved by the steepest descent method.

In nonlinear optimization problems, the calculation of the descent direction (perturbation) in parameter space is one of most intensive computation steps. Thus, its calculation needs to be very effective. In the steepest descent method, the descent direction is the gradient of the objective function (L2-norm of residuals). The descent direction can be calculated optimally by the adjoint method which estimates the gradient of objective function without Fréchet derivatives (Lailly, 1983; Tarantola, 1984; Plessix, 2006; Virieux and Operto, 2009).

To apply 1D source wavelet estimation by the adjoint method, it was used a 2D synthetic seismic dataset generated by the approximation of the $2 \mathrm{D}$ wave equation by finite differences in time domain (Levander, 1989). The Marmousi velocity model (figure 1a) was used to create the observed data. The source wavelet used to generate the observed data has a wide-band frequency. It's a relevant issue because the observed data was decomposed in different ranges of frequency.

The wavelet estimation algorithm used the trace closest of the source in each seismogram (observed data). The chosen trace was compared to the data calculated by the 1D wave equation modeling. The objective function was computed and used to calculate the gradient direction by the adjoint method. Then, it was solved the optimization problem iteratively through the steepest descent method.

The inverted wavelet was filtered in the same range of frequencies of the observed dataset and applied in the FWI process to estimate the velocity model from the initial velocity model (figure $1 \mathrm{~b}$ ). With this approach was possible to estimate the source wavelet in time domain multiscale FWI context. This methodology will permit to add the 


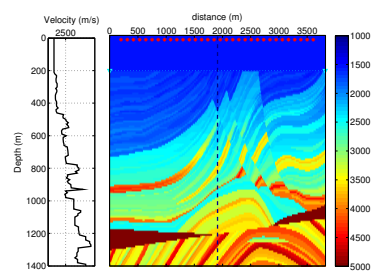

(a)
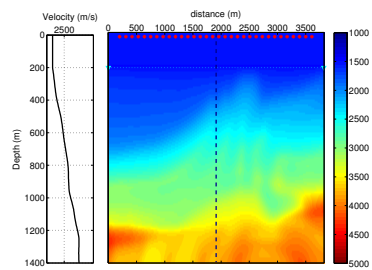

(b)

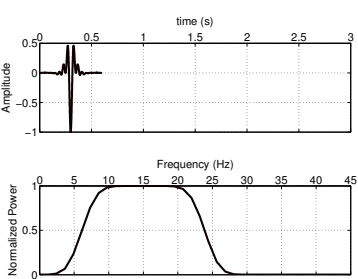

(a)

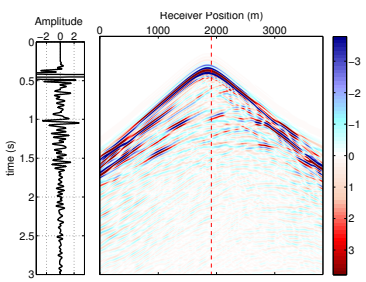

(b)
Figure 1: a) Marmousi acoustic velocity model [(Versteeg, 1994);(Martin et al., 2006)] b) Smoothed Marmousi velocity model, it was used as the initial model of the FWI workflow. The cyan dots represent the receiver positions in the sea bed and the red asterisks are the source positions. The black dashed line indicates the position of the profile on the left side.

wavelet inversion into the FWI workflow. In addition, the seismic inversion synthetic experiment will become more realistic.

\section{Materials and Methods}

\section{Synthetic Observed Data}

The observed data was generated synthetically using a source wavelet with a wide band of the frequency spectrum (figure 2a) to simulate a real seismic source. This source wavelet was calculated using the expression given by Cao and Han (2011). They called it Wide Band B-spline wavelet, and has the following expression:

$$
w\left(\mathbf{x}_{s}, t\right)=\frac{1}{q-p} \sqrt{f_{b}}\left(\operatorname{sinc}\left(\frac{f_{b} . t}{m}\right)\right)^{m}(q \operatorname{sinc}(2 q t)-p \operatorname{sinc}(2 p t)) \delta\left(\mathbf{x}-\mathbf{x}_{s}\right),
$$

where the parameters $q, p, f_{b}$ e $m$ define the source frequency spectrum with precision. And $\mathbf{x}$ is the 2D spacial coordinates ( $\mathrm{x}$ and $\mathrm{z}), \mathbf{x}_{s}$ is the source positions, $\delta$ is Dirac delta function and $t$ is the time. The parameters were chosen to generate a source wavelet with a wide frequency spectrum and cutoff frequency of $30 \mathrm{~Hz}$. The chosen parameters were $q=25, p=5, f_{b}=20$ e $m=8$.

The 2D acoustic wave equation:

$$
\frac{1}{c^{2}(\mathbf{x})} \frac{\partial^{2}}{\partial t^{2}} p(\mathbf{x}, t)-\nabla^{2} p(\mathbf{x}, t)=w\left(\mathbf{x}_{s}, t\right),
$$

and its discretization using finite difference of 4th order in space and 2nd order in time (Levander, 1989) was used to propagate the source wavelet (equation 1) through the velocity model $c(\mathbf{x})$. The pressure field $p(\mathbf{x}, t)$ was registered on sea bed $\left(d_{o b s}=p\left(\mathbf{x}_{r}, t\right)\right)$, where $\mathbf{x}_{r}$ is the receiver positions. This configuration simulates a seismic acquisition using a ocean bottom cable (OBC). The figure $2 \mathrm{~b}$ shows an example of synthetic observed data using Marmousi acoustic velocity model (figure 1a). It was generated 32 shots with 3 seconds of registering each shot.

\section{Data decomposition}

In pursuance of applying FWI in the time domain, the observed data was decomposed in different range of
Figure 2: a) Wide Band B-spline wavelet (Cao and Han, 2011) used to generated synthetic observed data. b) Synthetic seismogram created using Wide Band B-spline wavelet used as observed data. The red dashed line indicates the position of trace on the left.

frequencies to take advantage of the multiscale approach and avoid the cycle skipping problem (Virieux and Operto, 2009). For this reason, the observed data was filtered in some ranges of frequencies. In this work, we use the frequencies of $0-12 \mathrm{~Hz}, 0-18 \mathrm{~Hz}, 0-24 \mathrm{~Hz}$ and $0-30 \mathrm{~Hz}$ in the FWI.

The decomposition of the observed data in several ranges of frequencies can be done using a low pass filter. It was applied a zero-phase, sine-squared tapered filter. The observed data (figure $2 \mathrm{~b}$ ) was filtered with a cut frequency of $12 \mathrm{~Hz}, 18 \mathrm{~Hz}$ and $24 \mathrm{~Hz}$. Two examples of filtered Seismogram with cut frequency of $12 \mathrm{~Hz}$ and $24 \mathrm{~Hz}$ are showed in the figures $3 \mathrm{a}$ and $3 \mathrm{~b}$, respectively.

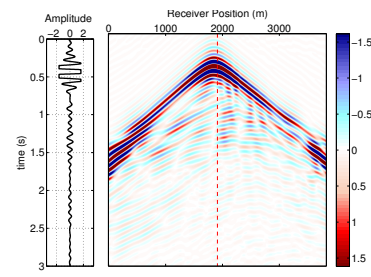

(a)

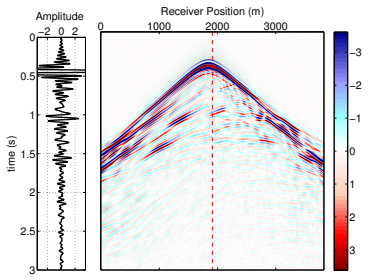

(b)
Figure 3: Low pass filter applied on observed data with cutoff frequency of a) $12 \mathrm{~Hz}$ and b) $24 \mathrm{~Hz}$. The red dashed line indicates the position of the trace on the left.

\section{Wavelet Estimation}

According to Schuster (2017), the source wavelet inversion can be viewed as 1D full waveform inversion (FWI). This nonlinear iterative inverse problem was solved by the steepest descent method (Nocedal and Wright, 2006) and the update direction was found through the adjoint method.

The adjoint method (Lailly, 1983; Tarantola, 1984; Plessix, 2006; Virieux and Operto, 2009; Fichtner, 2010) permitted to calculate the gradient of objective function (update direction) without evaluation of Fréchet derivatives. This step is one of the most intensive computations tasks in seismic inversion problem algorithm and it can be done efficiently with the adjoint method.

For the source wavelet estimation was used the 1D wave equation (equation 2 with $\mathbf{x}=z$ ) as the forward problem. The source wavelet $w$ was chosen as the parameter that 
will be optimized. Then, it was used L2 norm objective function:

$$
\chi(w)=\frac{1}{2} \int_{T}\left\|\mathbf{d}_{o b s}\left(z_{r e c}, t\right)-\mathbf{d}_{c a l}\left(z_{r e c}, t\right)\right\|^{2} d t,
$$

in which the observed synthetic data, generated with the $2 \mathrm{D}$ wave equation is compared to the calculated data generated with the 1D wave equation. The finite differences method also was used to solve 1D forward problem and obtaining the 1D calculated data (seismic trace).

The method of Lagrange multipliers was used to minimize the objective function (equation 3 ) and find its local minimum under the constraint of forward problem. If we write the forward problem operator as $F[p(z, t)]=w(t)$, the unconstrained Lagrangian is given by (Plessix, 2006):

$$
\begin{aligned}
\mathscr{L}\left(w, p, p^{\dagger}\right)= & \frac{1}{2} \int_{T}\left\|\mathbf{d}_{o b s}\left(z_{\text {rec }}, t\right)-\mathbf{d}_{\text {cal }}\left(z_{\text {rec }}, t\right)\right\|^{2} d t \\
& -\int_{T} p^{\dagger}[F(p(z, t))-w(t)] d t,
\end{aligned}
$$

where $p^{\dagger}$ is the Lagrange multipliers or adjoint field.

The gradient of Lagrangian, considering the source wavelet as parameter to be inverted, is:

$$
\nabla \mathscr{L}\left(w, p, p^{\dagger}\right)=\left(\frac{\partial \mathscr{L}}{\partial w}, \frac{\partial \mathscr{L}}{\partial p}, \frac{\partial \mathscr{L}}{\partial p^{\dagger}}\right) .
$$

As we only want to seek solutions in wavelet space (parameter space), we can impose:

$$
\frac{\partial \mathscr{L}}{\partial p^{\dagger}}=0
$$

and we found the constrain used to construct the unconstrained Lagrangian, the forward operator:

$$
\frac{1}{c^{2}(z)} \frac{\partial^{2}}{\partial t^{2}} p(z, t)-\nabla^{2} p(z, t)=w(z, t)
$$

Second, we can impose:

$$
\frac{\partial \mathscr{L}}{\partial p}=0
$$

and we found the backward operator:

$$
\frac{1}{c^{2}(z)} \frac{\partial^{2}}{\partial t^{2}} p^{\dagger}(z, t)-\nabla^{2} p^{\dagger}(z, t)=\Delta d .
$$

The adjoint field $p^{\dagger}$ need to be calculated in reverse time using the residual $(\Delta d)$ as source (Fichtner, 2010). Note that forward field $(p=p(z, t, w))$ and adjoint field $\left(p^{\dagger}=\right.$ $\left.p^{\dagger}(z, t, w)\right)$ also depend of the wavelet. So, using chain rule:

$$
\begin{aligned}
\nabla \mathscr{L}\left(w, p, p^{\dagger}\right) & =\frac{\partial \mathscr{L}\left(w, p(w), p^{\dagger}(w)\right)}{\partial w} \\
& =\frac{\partial \mathscr{L}}{\partial w}+\frac{\partial \mathscr{L}}{\partial p} \frac{\partial p}{\partial w}+\frac{\partial \mathscr{L}}{\partial p^{\dagger}} \frac{\partial p^{\dagger}}{\partial w}
\end{aligned}
$$

Since we supposed that $\frac{\partial \mathscr{L}}{\partial p}=0$ and $\frac{\partial \mathscr{L}}{\partial p^{\dagger}}=0$, we found the direction $h$ that minimize the objective function, or the Lagrangian unconstrained (Schuster, 2017):

$$
h=\nabla \mathscr{L}\left(w, p, p^{\dagger}\right)=\frac{\partial \mathscr{L}\left(w, p, p^{\dagger}\right)}{\partial w}=p^{\dagger}\left(z_{r e c}, t\right)
$$

The equation 11 gives the update direction of the iterative nonlinear optimization problem that can be solved through steepest descent method:

$$
w_{k+1}(t)=w_{k}-\alpha_{k} h_{k}
$$

where $\alpha_{k}$ is the step length found by steepest descent method (Nocedal and Wright, 2006).

\section{Practical issues}

For the sake of applying the source wavelet inversion in the $2 \mathrm{D}$ dataset is necessary some adjusts to improve the final results. First, we need to select the nearest receiver of the source for each shot. Then, to compare the chosen trace with the calculated data derived from the 1D wave equation. Second, it is required to pick the first arrival and use it only as observed data. Third, to choose an initial wavelet that produces a calculated data with similar phase to observed data. The initial wavelet applied in the inversion was a Ricker (Ryan, 1994).

The adjoint field $p^{\dagger}(z, t)$ was calculated using the same 1D wave equation operator by finite differences that solve the forward problem. However, the modeling need to be done in reverse time and the residual data $\left(\Delta d\left(z_{\text {rec }}, t\right)=\right.$ $\left.\mathbf{d}_{\text {obs }}\left(z_{\text {rec }}, t\right)-\mathbf{d}_{c a l}\left(z_{\text {rec }}, t\right)\right)$ as the source term. The registered adjoint field at receiver position, $p^{\dagger}\left(z=z_{\text {rec }}, t\right)$, is the update direction of nonlinear iterative optimization problem given by equation 11 .

After the steepest descent method to achieve the local minimum, the phase of the inverted source wavelet was forced to be zero and its amplitude was rescaled for each range of frequencies used in the FWI process. In that way, the calculated data derived from the $2 \mathrm{D}$ wave equation operator using the estimated wavelet has an amplitude similar to the observed data. This implies in a residual data that has a better convergence rate in FWI algorithm.

\section{Results}

\section{Calculated data $-1 D$ problem}

The estimated wavelet was obtained using the observed seismogram (figure $2 \mathrm{~b}$ ). The initial calculated data was derived from $1 \mathrm{D}$ wave equation operator, it is represented as the dashed gray line in figure $4 \mathrm{a}$. The observed data is the solid black line. And the final estimated data (with the cut frequency of $30 \mathrm{~Hz}$ ) is showed as a solid gray line. The figure $4 \mathrm{~b}$ shows the comparison between the filtered observed data and the calculated data for the cut frequencies of $12 \mathrm{~Hz}, 18 \mathrm{~Hz}$, and $24 \mathrm{~Hz}$, respectively. 


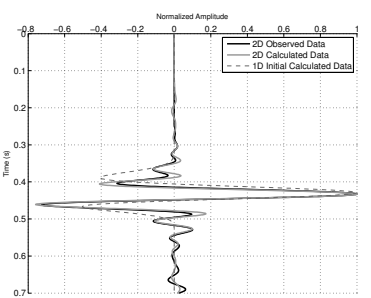

(a)

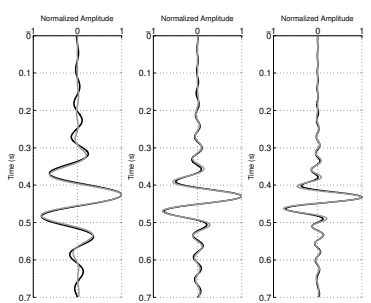

(b)

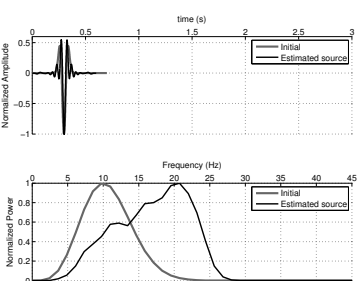

(a)
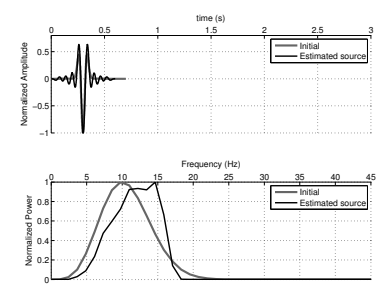

(c)
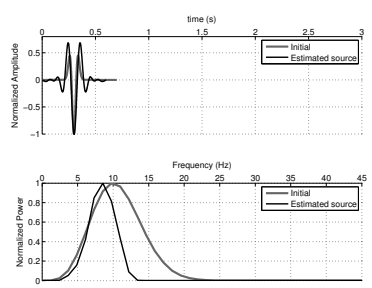

(b)
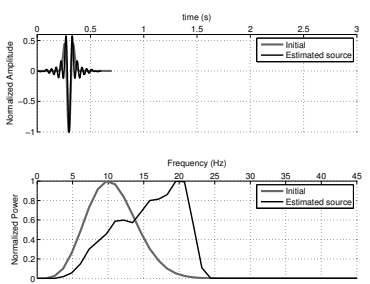

(d)

\section{Estimated source wavelet}

The initial source wavelet (gray lines) and estimated source wavelet (black lines) are showed in figure 5. On top of each figure are the normalized wavelets in the time domain. On the bottom, there are the normalized frequency spectrum of each wavelet. The initial wavelet chosen was the Ricker wavelet with the cut frequency of $30 \mathrm{~Hz}$.

In order to apply the multiscale strategy, the inverted wavelet (figure $5 \mathrm{a}$ ) was filtered using the same filter used in the observed data to decompose it the several frequencies. The same parameters was chosen to filter the estimated wavelet with cut frequencies of $12 \mathrm{~Hz}$ (figure $5 \mathrm{~b}$ ), $18 \mathrm{~Hz}$ (figure 5c) and $24 \mathrm{~Hz}$ (figure 5d).

\section{Calculated and residual data - 2D problem}

The calculated data (figure 6a) using the inverted wavelet and the 2D wave equation operator was compared to the synthetic observed data (figure $2 \mathrm{~b}$ ). It was used the same velocity model (figure 1a) to generate the calculated data. The residual is showed in figure $6 \mathrm{~b}$, it was calculated doing the difference between the observed and calculated data.

\section{Velocity model inversion}

The estimated wavelets (figure 5) were used in FWI process for each range of frequency of the multiscale approach $(0-12 \mathrm{~Hz}, 0-18 \mathrm{~Hz}, 0-24 \mathrm{~Hz}$ and $0-30 \mathrm{~Hz})$. The true velocity model (figure 1a) was smoothed and it was used as initial model (figure 1b) in the FWI. The initial velocity model mimics a low wavenumber velocity model constructed through a tomography method or using a welllog information. The inversion of the velocity model used the quasi-Newton I-BFGS optimization method (Métivier and Brossier, 2016) and the adjoint state method to find the update direction (objective function gradient) in the model space. The final results of the inversion for each frequency are shown in the Figure 7.

\section{Discussion and Conclusion}

We used a 2D synthetic seismic experiment to apply a seismic inversion method to estimate the source wavelet through the seismograms (figure $2 \mathrm{~b}$ ). The source wavelet estimation used the adjoint method (equation 11) to calculate the gradient directions and the steepest descent
Figure 5: Initial wavelet (gray lines) and inverted wavelet (black lines) for a) the inverted wavelet with cut frequency of $30 \mathrm{~Hz}$, b) filtered inverted wavelet with cut frequency of $12 \mathrm{~Hz}, \mathrm{c}$ ) filtered inverted wavelet with cut frequency of 18 $\mathrm{Hz}$ and d) filtered wavelet with cut frequency of $24 \mathrm{~Hz}$. On the upper part of the figures are the normalized wavelet in the time domain and on the bottom part of the figures are the normalized amplitude spectrum of the wavelets.

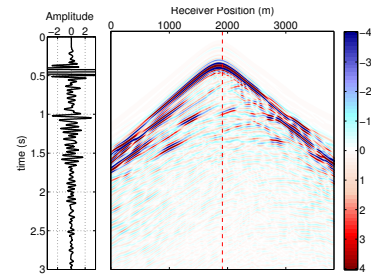

(a)

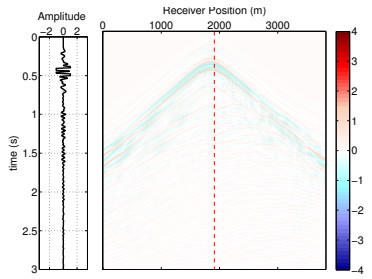

(b)
Figure 6: a) Example of a calculated data using the true model and the estimated wavelet. b)Residual between observed and calculated data.

method (equation 12) to solve the nonlinear optimization problem, the same methods used in FWI process.

The numerical experiment used the same theoretical ingredients to solve the direct and the inverse problem, this is known as the inverse crime (Wirgin, 2004). This procedure was done to avoid any seismic pre-processing of the observed data (Virieux and Operto, 2009; Sears et al., 2010; Ratcliffe et al., 2011).

An amplitude rescaling of the estimated source wavelet was done because of the differences between the 1D and 2D wave equation. The source wavelet estimation used the 1D wave equation operator while the synthetic observed dataset and the FWI used $2 \mathrm{D}$ wave equation operator. So, the amplitude of the source wavelet estimated was forced to generate a similar amplitude to the observed data. It is important to note that the rescaling was done for each range of frequency of the FWI process. Thus, the estimated wavelet (figure 5a) was filtered using the cut 


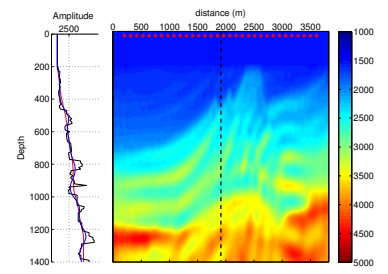

(a)

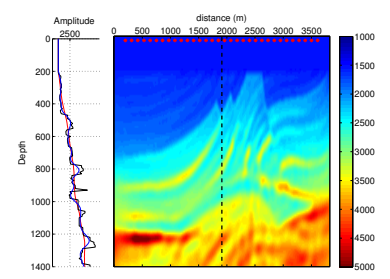

(c)

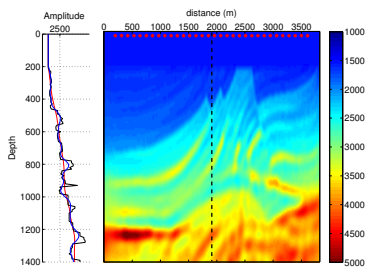

(b)
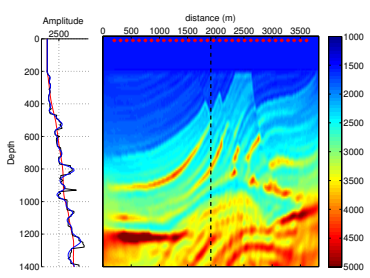

(d)
Figure 7: The velocity models inverted by the time domain FWI. It was applied to the multiscale approach using the source wavelet inverted for each cut frequency. a) $12 \mathrm{~Hz}$ b) $18 \mathrm{~Hz} \mathrm{c)} 24 \mathrm{~Hz}$ and d) $30 \mathrm{~Hz}$. The dashed black lines indicate the position of the profile on the left of each figure. On the profiles, the black lines are the true velocity model, the red lines are the initial velocity model and the blue lines are the estimated velocity model.

frequencies of $12 \mathrm{~Hz}$ (figure $5 \mathrm{~b}$ ), $18 \mathrm{~Hz}$ (figure $5 \mathrm{c}$ ) and 24 $\mathrm{Hz}$ (figure $5 \mathrm{~d}$ ). Those filtered wavelets was used as source wavelet in each step of the multiscale approach of FWI.

Despite the amplitude correction, the final result of the FWI process provided an inverted velocity model (figure 7d) similar to the true model (figure 1a). On the estimated model is possible to see the main geological structures suggesting that the estimated model achieved a minimum. The intermediate inverted models for each cut frequency (figures 7a, 7b and 7c) show the increase of the resolution for each step of multiscale approach.

The estimated source wavelet (figure $5 \mathrm{a}$ ) is very similar to the true wavelet (figure 2a) that was used to generate the observed data. The main difference can be seen in the frequency spectrum, where part of the low frequencies are missing. Despite that, the inverted wavelet generated a calculated data (figure 6a) very similar to the observed seismogram (figure $2 \mathrm{~b}$ ), providing small residuals (figure 6b).

Finally, this methodology can be used to make the synthetic seismic experiment more realistic in the time domain FWI context. In future works, we can include random noise to the observed dataset and try different acquisition geometries with the same propose. Furthermore, this methodology shows that is possible to integrate the source wavelet estimation using the adjoint method in FWI workflow.

\section{References}

Brossier, R., Operto, S., and Virieux, J., 2009, Seismic imaging of complex onshore structures by 2D elastic frequency-domain full-waveform inversion: Geophysics, 74, no. 6, WCC105-WCC118.
Bunks, C., Saleck, F. M., Zaleski, S., and Chavent, G., 1995, Multiscale seismic waveform inversion: Geophysics, 60, no. 5, 1457-1473.

Cao, S., and Han, D., 2011, Wide-band b-spline wavelet with four parameters: SEG Technical Program Expanded Abstracts 2011, pages 3840-3844.

Fichtner, A., 2010, Full seismic waveform modelling and inversion: Springer Science \& Business Media.

Lailly, P., 1983, The Seismic Inverse Problem as a Sequence of Before Stack Migrations: Conference on Inverse Scattering, Theory and Applications, Society for Industrial and Applied Mathematics, pages 206-220.

Levander, A., 01 1989, Finite-difference forward modeling in seismology: Encyclopedia of Solid Earth Geophysics, pages 410-431.

Martin, G. S., Wiley, R., and Marfurt, K. J., 2006, Marmousi2: An elastic upgrade for marmousi: The Leading Edge, 25, no. 2, 156-166.

Métivier, L., and Brossier, R., 2016, The seiscope optimization toolbox: A large-scale nonlinear optimization library based on reverse communicationthe seiscope optimization toolbox: Geophysics, 81, no. 2, F1-F15.

Nocedal, J., and Wright, S., 2006, Numerical optimization:, Springer Series in Operations Research and Financial Engineering Springer New York.

Plessix, R.-E., 2006, A review of the adjoint-state method for computing the gradient of a functional with geophysical applications: Geophysical Journal International, 167, no. 2, 495-503.

Pratt, R. G., 1999, Seismic waveform inversion in the frequency domain, part 1: Theory and verification in a physical scale model: Geophysics, 64, no. 3, 888-901.

Ratcliffe, A., Win, C., Vinje, V., Cggveritas, G. C., Warner, M., Umpleby, A., Stekl, I., Nangoo, T., College, I., and Conocophillips, A. B., 2011, Full Waveform Inversion : a North Sea OBC case study: SEG San Antonio 2011 Annual Meeting, pages 2384-2388.

Ryan, H., 1994, Ricker, Ormsby; Klander, Butterworth -A Choice of wavelets: CSEG Recorder, September, 2425.

Schuster, G., 2017, Seismic inversion: Society of Exploration Geophysicists.

Sears, T. J., Barton, P. J., and Singh, S. C., 2010, Elastic full waveform inversion of multicomponent ocean-bottom cable seismic data: Application to Alba Field, U. K. North Sea: Geophysics, 75, no. 6, R109-R119.

Tarantola, A., 1984, Inversion of seismic reflection data in the acoustic approximation: Geophysics, 49, no. 8, 1259-1266.

Versteeg, R., 1994, The Marmousi experience: Velocity model determination on a synthetic complex data set: The Leading Edge, 13, no. 9, 927-936. 
Virieux, J., and Operto, S., 2009, An overview of full-waveform inversion in exploration geophysics: Geophysics, 74, no. 6, WCC1-WCC26.

Wang, Y., 2016, Seismic Inversion: Theory and Applications:.

Wirgin, A., The inverse crime:, 2004.

\section{Acknowledgments}

I would like to thanks the CAPES to finance the research, the UFF and the GISIS by the infrastructure and support, the PGS by the donation of the computer cluster used in the numeric experiments and the GISIS's colleagues, in special, to the Rodrigo Stern by the IT support and the Oscar by the revision of the text. 